\section{Response to Preiksaitis and Limaye}

We thank Preiksaitis and Limaye for their comments on our paper ${ }^{1}$ and their interest and we attempt to provide herewith responses to their questions. First, they correctly note that we did not find an association between viral infection and acute rejection in our lung transplant recipient (LTR) cohort. ${ }^{2}$ However, this finding does not preclude a possible association between viral infection and bronchiolitis obliterans syndrome (BOS). While the temporal association between viral infection and acute rejection is expected to be short, the BOS inflammatory process and lung function decline may develop months after infection. In our study, follow-up was too short in many LTRs at this stage to allow for an adequate analysis of factors associated with incident BOS. Analyses pertaining to such are currently ongoing.

We agree with Preiksaitis and Limaye that respiratory viral infections have been under-recognised thus far. They raise the criticism that surveillance testing was too infrequent to capture all viral infections in our LTR cohort and thus underestimates their true prevalence. In our study, mean prevalence of asymptomatic viral carriage was $10 \%$ (range 2.3-20.0\%) during screening periods. During the period of highest prevalence $(20 \%)$ in autumn of 2008, these asymptomatic infections were due exclusively to picornavirus infection. This is not surprising since LTR with picornavirus infections did report fewer new symptoms (mean sum 2.2) compared with those with other viruses. Broadly speaking, beyond epidemics, the incidence of detected viral infection in a population is dependent on the sensitivity of the diagnostic test itself (eg, reverse-transcriptase PCR is highly sensitive) and the frequency of its use. Thus, by virtue of chance alone or a lead-time bias-like effect, more frequent reverse-transcriptase PCR screening may identify a higher prevalence of asymptomatic viral carriage. In our opinion, it seems likely that the prevalence of asymptomatic carriage will increase if more intensive screening is applied; whether this would exceed an annual mean of $10 \%$ is hypothetical and would certainly depend on seasonal effect. The high rate of asymptomatic carriage $(20 \%)$ found in our study, in autumn 2008, during a unique rhinovirus epidemic could serve as an example.

Preiksaitis and Limaye invite us to detail further the use of bronchoscopy in our study. Bronchoscopy with bronchoalveolar lavage $(\mathrm{BAL})(\mathrm{n}=276)$ was performed for usual care surveillance during screening periods (19.2\%) as part of routine post-transplant follow-up outside of screening periods $(43.5 \%)$ or when clinically indicated in the presence of new respiratory symptoms or worsening lung function (37.3\%). For ethical reasons, the study protocol did not include bronchoscopy without a clinical indication. Viral positivity rates of BAL specimens upon screening visits, routine visits and emergency visits were 7.5\% (4/ 53), 5.8\% (7/120) and 22.3\% (23/103), respectively.

We agree with Preiksaitis and Limaye that outcomes such as the incidence of subsequent bacterial or fungal infections as well as chronic allograft dysfunction should be studied in LTR with (and without) viral infections. We think that the type of virus and the severity of associated acute respiratory symptoms in this population should be analysed together as predictors of BOS. This implies that 
symptom severity scores should be developed and validated to address the wide range of symptom severity related to viral infection.

Finally, Preiksaitis and Limaye comment on the connection between viral infection of the upper and lower respiratory tracts. It is classically accepted in the field that most respiratory viruses begin by infecting the nasopharyngeal mucosa either via infected droplets or direct inoculation before spreading to the lower respiratory tract. Direct infection of the lower respiratory tract without any viral replication in the upper respiratory tract would imply aerosol transmission. In a previous study, we showed that a positive BAL together with a negative upper respiratory screening was unusual in the case of picornavirus infection. ${ }^{3}$ (This may not hold true for other viruses such as respiratory syncytial virus or influenza, which are indeed more likely to infect the lower respiratory tract directly.) But the classic pathogenetic model is being challenged by recent observations. For example, during the 2009 H1N1 outbreak, cases of severe pneumonia with negative nasopharyngeal screening were diagnosed. ${ }^{4}$ Similar examples have been described for the new coronavirus Middle-East Respiratory Syndrome. ${ }^{5} \quad$ Altogether, recent data on respiratory virus epidemiology suggest that the classic temporal connection between viral infection of the upper and lower respiratory tracts should be reconsidered.

Pierre-Olivier Bridevaux, ${ }^{1}$ J-D Aubert, ${ }^{2}$

${ }^{1}$ Division of Pulmonary Medicine, University Hospitals of Geneva, Geneva, Switzerland

${ }^{2}$ Division of Pulmonary Medicine, University Hospital of Lausanne, Lausanne, Switzerland

${ }^{3}$ Laboratory of Virology, Division of Infectious Diseases and Division of Laboratory Medicine, University Hospitals of Geneva and Medical School, University of Geneva, Geneva, Switzerland

Correspondence to Dr Pierre-Olivier Bridevaux, Division of Pulmonary Medicine, University Hospitals of Geneva, Geneva 1211, Switzerland; pierre-olivier. bridevaux@hcuge.ch

Contributors $\mathrm{P}-\mathrm{OB}$, J-DA and LK drafted the response which was amended and finally approved by $L N$ and $T R$.

Competing interests None.

Provenance and peer review Not commissioned; internally peer reviewed.

To cite Bridevaux P-O, Aubert J-D, Rochat $\mathrm{T}$, et al. Thorax 2014;69:82-83.

Received 30 October 2013
Accepted 30 October 2013

\section{SLinked}

http://dx.doi.org/10.1136/thoraxjnl-2013-204610

Thorax 2014;69:82-83.

doi:10.1136/thoraxjnl-2013-204768

\section{REFERENCES}

1 Preiksaitis CM, Limaye AP, Correspondence on the paper by Bridevaux, et al. Thorax 2014;69:82.

2 Bridevaux PO, Aubert JD, Soccal PM, et al. Incidence and outcomes of respiratory viral infections in lung transplant recipients: a prospective study. Thorax 2014;69:32-8.

3 Soccal PM, Aubert JD, Bridevaux PO, et al. Upper and lower respiratory tract viral infections and acute graft rejection in lung transplant recipients. Clin Infect Dis 2010;51:163-70.

4 Yeh E, Luo RF, Dyner L, et al. Preferential lower respiratory tract infection in swine-origin $2009 \mathrm{~A}$ (H1N1) influenza. Clin Infect Dis 2010;50:391-4.

5 Guery B, Poissy J, el Mansouf L, et al. Clinical features and viral diagnosis of two cases of infection with Middle East Respiratory Syndrome coronavirus: a report of nosocomial transmission. Lancet 2013;381:2265-72. 